\title{
The Mechanism Of Functional Electrical Stimulation Ameliorating Muscle Function Of Patients With Sarcopenia After Spinal Cord Injury: A Bioinformatics Analysis Of Key Genes
}

Kecheng Lao

Qingdao Municipal Hospital Group

Lixia Wang

Qingdao Municipal Hospital Group

Lihua Wang

Fujian Medical University

Xiao Fan ( $\square$ fanxiao_2001@163.com )

Qingdao Municipal Hospital Group https://orcid.org/0000-0001-5147-6701

Hailei Yin

No.971 hospital of PLA

\section{Research Article}

Keywords: functional electrical stimulation, spinal cord injury, sarcopenia, bioinformatics

Posted Date: April 28th, 2021

DOl: https://doi.org/10.21203/rs.3.rs-454491/v1

License: (c) (1) This work is licensed under a Creative Commons Attribution 4.0 International License.

Read Full License 


\section{Abstract}

Background: Sarcopenia caused by spinal cord injury seriously affects the muscle function, which impairs the locomotion function of patients. As an effective physiotherapeutic, functional electrical stimulation is benefit to the recovery of muscle function without exact mechanisms. So the objection of the study is to explore the biological regulatory factors related to functional electrical stimulation for muscle function recovery of patients with sarcopenia after spinal cord injury using bioinformatics methods.

Methods: The related microarray datasets GSE142426 and GSE33886 were downloaded from the Gene Expression Omnibus database. We used the R software to merge the two datasets, correct the inter-batch differences and screen the differentially expressed genes. Gene ontology enrichment analysis and Kyoto Encyclopedia of Genes and Genomes pathway were analyzed by using the DAVID online tool. The STRING database was used to analyze the interaction of differentially encoded proteins. The key genes were selected to draw the ROC curves, and CYCS was used to perform gene set enrichment analysis.

Results: A total of 114 differentially expressed genes were selected, including 44 up-regulated genes and 70 down-regulated genes, and four hub genes were identified including CYCS, SUCLG1, ATP5B and ATP5C1. ROC curve showed that CYCS was considered as the best indicator and GSEA analysis showed that up-regulation of CYCS was related to mitochondria and energy metabolism.

Conclusion: The mechanism of function electrical stimulation on muscle function recovery of spinal cord injury patients with sarcopenia is mainly related to regulate mitochondrial energy metabolism and scavenge reactive oxygen species to mitigate the oxidative damage.

\section{Introduction}

Spinal cord injury (SCl) means that damage to the spinal cord with temporarily or permanently in its functions. $\mathrm{SCl}$ with a high mortality and disability rate cause great damage to the survivors who lose the ability to work and live independently, meanwhile it can bring enormous burden to the physical, psychological, economic and family of the suffers. More than 27 million patients are suffering $\mathrm{SCl}$ around the word, and the incidence is rising year on year (1). Sarcopenia is a progressive and systemic skeletal muscle disorders with the accelerated loss of muscle mass and function and which can increased the risk of falls, fractures, disability and mortality $(2,3)$. Restricted mobility in SCI patients can cause the reduction of the cross-sectional area (CSA) of somatic muscles and increase the intramuscular lipid content, leading to the development of sarcopenia, and the rate of sarcopenia and weakness as high as $31.1 \%$ (4). Subsequently, SCl can induce impaired glucose tolerance, insulin resistance, and lipid abnormalities(5). A wealth of studies confirms that the functional electrical stimulation (FES) is contribute to the increase of muscle CSA, muscle strength and down-regulated the bone turnover, which is benefit to the recovery of muscle function(6-8). So far, the mechanism of FES treating SCI remains incompletely clear. Some studies suggest it is associated with mitochondrial function and energy 
metabolism. With the development of next-generation sequencing technologies and the improvement of biological database, using bioinformatics methods to explore the relevant mechanisms is significant.

\section{Methods}

Microarray studies and datasets from Gene Expression Omnibus (GEO)

The microarray datasets including GSE142426 and GSE33886 were downloaded from the GEO database (https://www.ncbi.nlm.nih.gov/geo/) using "spinal cord injury" as the keyword. The microarray dataset GSE142426 from GPL5175 platform contains 15 samples of male patients with SCI before and after treated with FES. The microarray dataset GSE33886 from GPL6244 platform contains 3 samples of male patients with $\mathrm{SCl}$ before and after treated with FES. Samples were selected by hierarchical clustering and all the samples were taken from quadriceps femoris or soleus.

Differential genes expression analysis

Statistical programming language $\mathrm{R}$ (version 4.0.2) was used for log2 transformation of the data, and the two datasets were merged. "SVA" package was used for batch correction. Differential expressed genes (DEGs) were defined as $\log |F C|>0.5$, and the corrected $p<0.05$. Log $|F C|>0$ means that the DEG is up regulated after FES treatment.

Functional annotation and pathway analysis of DEGs

DEGs were inputted into David 6.8 online tools(https://david.ncifcrf.gov/)to perform Gene Ontology (G0) analysis and Kyoto Encyclopedia of Genes and Genomes (KEGG) pathway enrichment. $P<0.05$ and the gene counts $>3$ were considered statistically significant.

Protein-protein interaction (PPI) network and key genes acquisition

Using the Search Tool for the Retrieval of Interacting Genes (STRING, version 11.0, https://string-db.org/) database to analyze the PPI of proteins encoded by DEGs (medium confidence $=0.04$ ). Cytoscape software (version 3.8.0) was used to perform visualization of PPI network. We used cytohubba plug-in to analyze the nodes of the genes with topological analysis methods, filtering with degree and stress and obtaining the key genes from the intersection of the first 15 genes sorted out in degree and stress methods respectively.

Receiver operating characteristic (ROC) curve analysis

Using the Graphpad prism (version 8.2.1) software to draw the ROC curve and the area under curve (AUC) was used to evaluate the sensitivity and specificity of candidates with the AUC more than 0.7. According to the Youden index, the best cut-off value was selected and $\mathrm{P}<0.05$ means the difference was statistically significant.

Gene set enrichment analysis (GSEA)

We choose the CYCS gene which is highest ranked in degree method and statistically significant in ROC curve to perform GSEA analysis. The gene expressions were divided into low expression group and high 
expression group according to the median. We analyzed the KEGG pathway related to CYCS high expressions filtering with P $₫ 0.05$, NES $₫ 1$ and FDR $₫ 25 \%$ and using David analysis method to obtain the key pathway from the intersection of the two analyses.

\section{Results}

Hierarchical clustering for sample selection

The total samples were analyzed by hierarchical clustering, and 2 samples (GSM4227252 and GSM4227238) with high heterogeneity were eliminated. Finally, 17 samples were included before and after FES.

Identification of DEGs

A total of 114 DEGs were screened out, including 44 high expression genes and 70 low expression genes. $\mathrm{R}$ was used to make results visualization and draw volcano map and heat map (Fig. 1).

\section{GO and KEGG enrichment analysis of DEGs}

In GO analysis, DEGs were divided into three categories: biological process, cellular component and molecular function. In the biological process, DEGs were mainly concentrated in the fields of mitochondrial electrical transport, NADH to ubiquinone, mitochondrial respiratory chain complex I assembly, mitochondrial adenosine triphosphate (ATP) synthesis coupled proton transport, tricarboxylic acid cycle, response to reactive oxygen species, etc. In the cellular components, DEGs were mainly concentrated in the parts of mitochondrial inner membrane, mitochondrion, mitochondrial matrix, mitochondrial respiratory chain complex I, extracellular exosome and so on. In molecular functions, DEGs were mainly concentrated in poly (A) RNA binding, NADH dehydrogenase activity, electron carrier activity, NADH dehydrogenase (ubiquinone) activity, proton-transporting ATP synthase activity, rotational mechanism and so on. The above-mentioned results show that the difference genes mainly focus on mitochondrial function and energy metabolism. KEGG pathway analysis shows that DEGs mainly focuses on pathways about Alzheimer's disease, Parkinson's disease, Oxidative phosphorylation, Huntington's disease, Non-alcoholic fatty liver disease (NAFLD), Metabolic pathways, tricarboxylic acid (TCA) cycle, Carbon metabolism and so on, which can be seen as the main pathways involved in nervous system diseases and energy metabolism (Fig. 2).

PPI network analysis of DEGs

A total of 113 nodes and 363 pairs of protein interaction relationships were obtained. After eliminating independent genes, Cytoscape was used for visualization of PPI network map and 4 key genes including CYCS, SUCLG1, ATP5B and ATP5C1 were obtained from the intersection of Wayne map (Fig. 3).

Evaluation of diagnostic value of hub gene with ROC curve analysis ROC curve was drawn for 4 hub genes to evaluate the effectiveness of hub genes of $\mathrm{SCl}$ patients after FES. CYCS (AUC $=0.7578, P=0.0103$ ) was considered as the best indicator (Table 1, Fig. 4). 
Table 1

Evaluation of key genes diagnostic capabilities

\begin{tabular}{|lllll|}
\hline Gene & Sensitivity\% & Specificity\% & AUC & P Value \\
\hline ATP5C1 & 47.06 & 88.24 & 0.6574 & 0.1171 \\
\hline SUCLG1 & 88.24 & 47.06 & 0.6747 & 0.0820 \\
\hline ATP5B & 88.24 & 52.94 & 0.6955 & 0.0516 \\
\hline CYCS & 94.12 & 52.94 & 0.7578 & 0.0103 \\
\hline \multicolumn{4}{|l}{ Abbreviation: AUC, area under curve } & \\
\hline
\end{tabular}

GSEA of CYCS gene

To clarify the mechanism of effectiveness of CYCS gene on patients with SCI after FES, we performed the GSEA. According to the adjusted expression level of CYCS, the samples were divided into high and low expression groups. The results show that high CYCS expression samples were mainly enriched in cardiac muscle contraction, Parkinson's disease, Alzheimer's disease, oxidative phosphorylation, TCA cycle, etc. (Fig. 5).

\section{Discussion}

As a serious central nervous system injury, the impact of $\mathrm{SCl}$ on patients is inestimable. The effects of FES, an important adjuvant therapy on $\mathrm{SCl}$, on Sarcopenia and weakness in $\mathrm{SCl}$ patients have been confirmed by many studies. But the exact mechanism of the effects of FES is not known. It has been reported in previous studies that mitochondria and energy metabolism play an important role in this process, which has been confirmed again in our study.

Through PPI network, we identified four genes including CYCS, SUCLG1, ATP5B and ATP5C1, which may be the key genes for FES to improve muscle function in patients with SCI. Around these genes, CYCS has been suggested to be closely related to the improvement of muscle function. Previous studies have confirmed that the reduction of exercise after $\mathrm{SCl}$ can reduce the ratio of fusion proteins and lead to mitochondrial dysfunction $(9,10)$, while exercise training can improve the volume, quantity, density, dynamics and quality control of skeletal muscle mitochondrial $(11,12)$.

As an indispensable component in the oxidative respiratory chain, CYCS is the core component of mitochondrial electron transport chain and the proteins encoded by CYCS combines with the mitochondrial inner membrane to act as the electronic carrier of complexes $\nabla$ to complex $\nabla$ in the electron transport chain(13). Oxidative stress caused by long-term inactivity plays an important role in muscle atrophy, which can reduce mitochondrial respiratory function. The main source of oxidants is reactive oxygen species (ROS) produced by mitochondria and high concentration of ROS can damage fast and slow muscle fibers and mitochondrial function, and lead to muscle fiber atrophy(14). CYCS can clear ROS and reduce the damage caused by oxidative stress to improve muscle function $(15,16)$. At the same time, exercise can increase the abundance of mitochondrial RNA and the ability of mitochondria on producing 
ATP (17). SUCLG1 is mainly composed of the catalytic enzyme of mitochondrial energy generation. ATP5B and ATP5C1 are mainly involved in the subunit of ATP syntheses in mitochondria, and the three genes participate in promoting energy generation. Therefore, FES may improve muscle quality and strength by promoting the production of the above four genes.

We analyzed the CYCS gene that is highest ranked in degree method by GSEA combined with the enrichment of KEGG pathway in DAVID analysis. The results showed that the up regulation of CYCS was related to myocardial contraction, Parkinson's disease, Alzheimer's disease, oxidative phosphorylation and TCA cycle. It is confirmed that CYCS is closely related to energy metabolism such as oxidative phosphorylation, TCA cycle and so on. $95 \%$ of the energy of cell activity comes from ATP produced by oxidative phosphorylation (18). Oxidative phosphorylation is composed of electron transport chain and ATP synthase. The former generates mitochondrial proton power by pumping protons across the inner mitochondrial membrane and the latter returns protons to the matrix to couple with ADP and phosphate to ATP (18). One of the main protein control the signal of mitochondria oxidative phosphorylation is cytochrome $c$, and the function of which was regulated by phosphorylation of several residues $(18,19)$. So, as the final step of electron transport chain, CYCS is an important place for the oxidation phosphorylation of electron transport chain. The TCA cycle is the final metabolic pathway of the three major nutrients as lipids, carbohydrates, and amino acids, and is the main site for energy metabolism in the mitochondria. So, as the metabolic link of three substances, TCA cycle is the key link of energy metabolism and the main place is mitochondria. Therefore, all the above evidence supports that FES is associated with mitochondria and energy metabolism.

The results of GO enrichment analysis suggests that the DEGs mainly focus on mitochondrial electron transport, mitochondrial respiratory chain complex I assembly, mitochondrial ATP synthesis coupled proton transport, TCA cycle, response to reactive oxygen species and so on. These results are consistent with our differential analysis and KEGG pathway, which mainly focus on mitochondrial energy metabolism.

As muscle samples are more difficult to obtain, bioinformatics methods were used for DEGs analysis as a favorable tool to explore the mechanism of FES on muscle function recovery of $\mathrm{SCl}$ patients. But due to the small sample size of these two datasets, some causative genes may not show significant differences and we selected a smaller difference. As the number of datasets in the GEO database increasing and relevant techniques and algorithm improved, the mechanisms about FES on treating SCI patients may be further explored.

\section{Conclusions}

The mechanism of FES on muscle function recovery of spinal cord injury patients with sarcopenia is mainly related to regulate mitochondrial energy metabolism and scavenge reactive oxygen species to mitigate the oxidative damage. 


\section{Abbreviations}

SCl: spinal cord injury; CSA: cross-sectional area; FES: functional electrical stimulation; GEO: gene expression omnibus, DEGs: differential expressed genes; GO: gene ontology; KEGG: Kyoto Encyclopedia of Genes and Genomes; PPI: protein-protein interaction; STRING: search tool for the retrieval of interacting genes; ROC: receiver operating characteristic; AUC: area under curve; GSEA: gene set enrichment analysis; TCA: tricarboxylic acid; adenosine triphosphate: ATP.

\section{Declarations}

Ethics approval and consent to participate: Not applicable.

Consent for publication: Not applicable.

Availability of data and materials: The datasets used and/or analyzed during the current study are available from the corresponding author on reasonable request.

Competing interests: The authors declare that they have no competing interests.

Funding: This work was supported by Shandong province science and technology development plan of traditional Chinese medicine (No. 2019-0614) and Fujian universities and colleges engineering research center of marine biopharmaceutical resources (No. XMMC-MBS201903).

Authors' contributions: KCL designed the study and prepared the manuscript. LXW and LHW searched database and extracted datasets. XF and HLY as the co- corresponding authors designed the study and analyzed the datasets.

Acknowledgements: Not applicable.

\section{References}

1. Nadel J, McNally JS, DiGiorgio A, Grandhi R. Emerging Utility of Applied Magnetic Resonance Imaging in the Management of Traumatic Brain Injury. Medical sciences (Basel, Switzerland). $2021 ; 9(1)$.

2. Cruz-Jentoft AJ, Sayer AA. Sarcopenia. Lancet. 2019;393(10191):2636-46.

3. Cruz-Jentoft AJ, Bahat G, Bauer J, Boirie Y, Bruyere O, Cederholm T, et al. Sarcopenia: revised European consensus on definition and diagnosis. Age Ageing. 2019;48(1):16-31.

4. Rodriguez G, Berri M, Lin P, Kamdar N, Mahmoudi E, Peterson MD. Musculoskeletal morbidity following spinal cord injury: A longitudinal cohort study of privately-insured beneficiaries. Bone. 2021;142:115700.

5. Gorgey AS, Dolbow DR, Dolbow JD, Khalil RK, Castillo C, Gater DR. Effects of spinal cord injury on body composition and metabolic profile - part I. J Spinal Cord Med. 2014;37(6):693-702. 
6. Gargiulo P, Reynisson PJ, Helgason B, Kern H, Mayr W, Ingvarsson P, et al. Muscle, tendons, and bone: structural changes during denervation and FES treatment. Neurol Res. 2011;33(7):750-8.

7. Johnston TE, Marino RJ, Oleson CV, Schmidt-Read M, Leiby BE, Sendecki J, et al. Musculoskeletal Effects of 2 Functional Electrical Stimulation Cycling Paradigms Conducted at Different Cadences for People With Spinal Cord Injury: A Pilot Study. Arch Phys Med Rehabil. 2016;97(9):1413-22.

8. Kern H, Carraro U, Adami N, Biral D, Hofer C, Forstner C, et al. Home-based functional electrical stimulation rescues permanently denervated muscles in paraplegic patients with complete lower motor neuron lesion. Neurorehabil Neural Repair. 2010;24(8):709-21.

9. Iqbal S, Ostojic O, Singh K, Joseph AM, Hood DA. Expression of mitochondrial fission and fusion regulatory proteins in skeletal muscle during chronic use and disuse. Muscle Nerve. 2013;48(6):96370.

10. McCully KK, Mulcahy TK, Ryan TE, Zhao Q. Skeletal muscle metabolism in individuals with spinal cord injury. J Appl Physiol (1985). 2011;111(1):143-8.

11. Axelrod CL, Fealy CE, Mulya A, Kirwan JP. Exercise training remodels human skeletal muscle mitochondrial fission and fusion machinery towards a pro-elongation phenotype. Acta Physiol (Oxf). 2019;225(4):e13216.

12. Holloszy JO, Coyle EF. Adaptations of skeletal muscle to endurance exercise and their metabolic consequences. J Appl Physiol Respir Environ Exerc Physiol. 1984;56(4):831-8.

13. Kalpage HA, Bazylianska V, Recanati MA, Fite A, Liu J, Wan J, et al. Tissue-specific regulation of cytochrome $\mathrm{c}$ by post-translational modifications: respiration, the mitochondrial membrane potential, ROS, and apoptosis. FASEB journal: official publication of the Federation of American Societies for Experimental Biology. 2019;33(2):1540-53.

14. Min K, Smuder AJ, Kwon OS, Kavazis AN, Szeto HH, Powers SK. Mitochondrial-targeted antioxidants protect skeletal muscle against immobilization-induced muscle atrophy. J Appl Physiol (1985). 2011;111(5):1459-66.

15. Li S, Sun C, Gu Y, Gao X, Zhao Y, Yuan Y, et al. Mutation of IDH1 aggravates the fatty acidinduced oxidative stress in HCT116 cells by affecting the mitochondrial respiratory chain. Mol Med Rep. 2019;19(4):2509-18.

16. Giorgio M, Migliaccio E, Orsini F, Paolucci D, Moroni M, Contursi C, et al. Electron transfer between cytochrome $\mathrm{c}$ and p66Shc generates reactive oxygen species that trigger mitochondrial apoptosis. Cell. 2005;122(2):221-33.

17. Lanza IR, Short DK, Short KR, Raghavakaimal S, Basu R, Joyner MJ, et al. Endurance exercise as a countermeasure for aging. Diabetes. 2008;57(11):2933-42.

18. Huttemann M, Lee I, Grossman LI, Doan JW, Sanderson TH. Phosphorylation of mammalian cytochrome $\mathrm{c}$ and cytochrome $\mathrm{c}$ oxidase in the regulation of cell destiny: respiration, apoptosis, and human disease. Adv Exp Med Biol. 2012;748:237-64.

19. Moreno-Beltran B, Guerra-Castellano A, Diaz-Quintana A, Del Conte R, Garcia-Maurino SM, DiazMoreno S, et al. Structural basis of mitochondrial dysfunction in response to cytochrome C 
phosphorylation at tyrosine 48. Proc Natl Acad Sci U S A. 2017;114(15):E3041-E50.

\section{Figures}

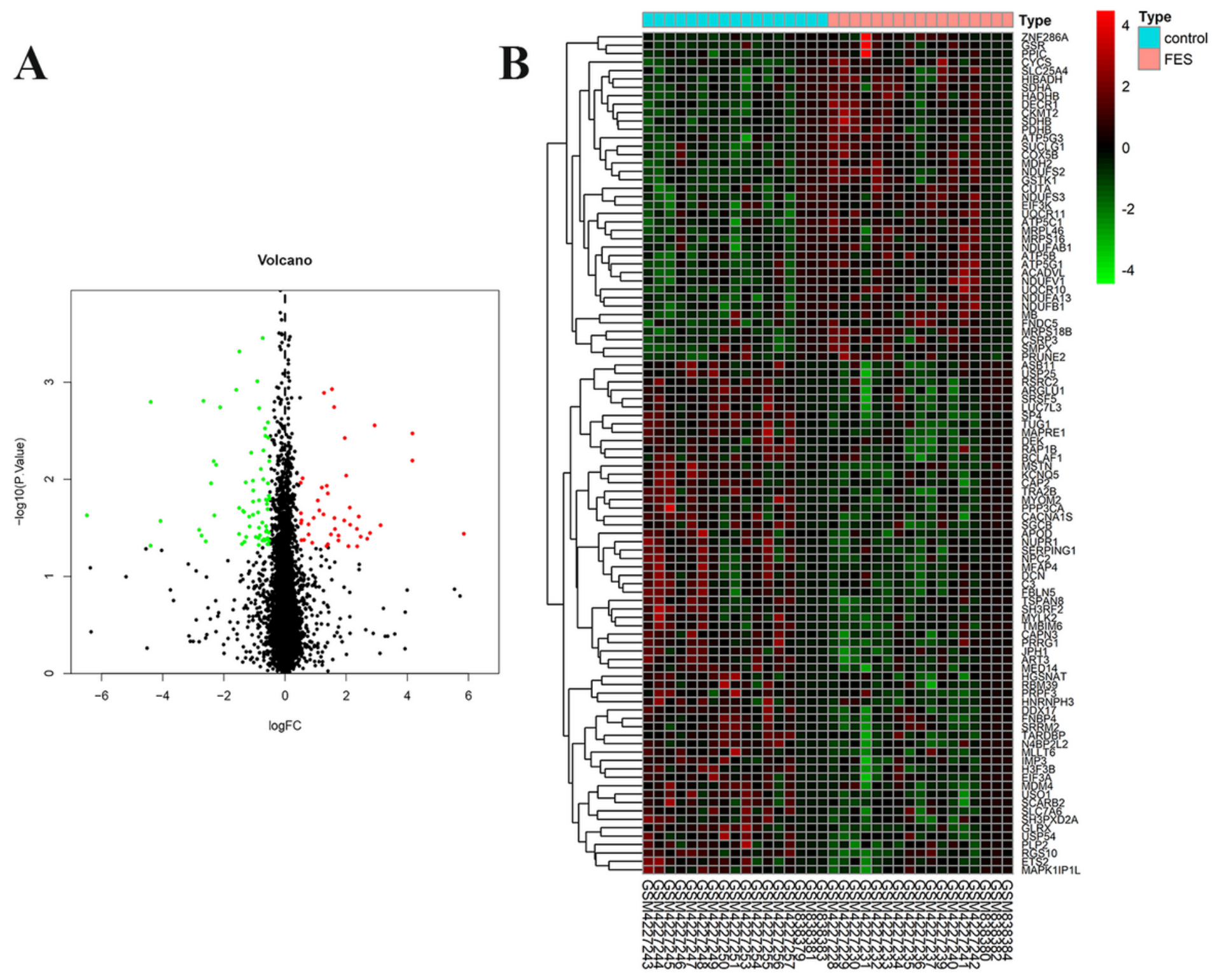

Figure 1

Volcano plot, cluster dendrogram and heat map of differentially expressed genes (A) The volcano showed that differentially expression genes identified from both GSE142426 and GSE33886. Red dots indicate up-regulated genes screened according to $\| \log \mathrm{FCl}>0.5$ and P-value $<0.05$, and green dots indicate downregulated genes screened according to $|\log \mathrm{FC}|>0.5$ and P-value $<0.05$. Black dots represent expression level of genes without statistically significant. (B) Heat maps show the top 100 differentially expression genes, the horizontal ordinate represent the names of the samples in the microarray and the vertical ordinate represent the names of the differential genes. Red represents high expression, green represents low expression and black represents non-significant relative expression differences. 


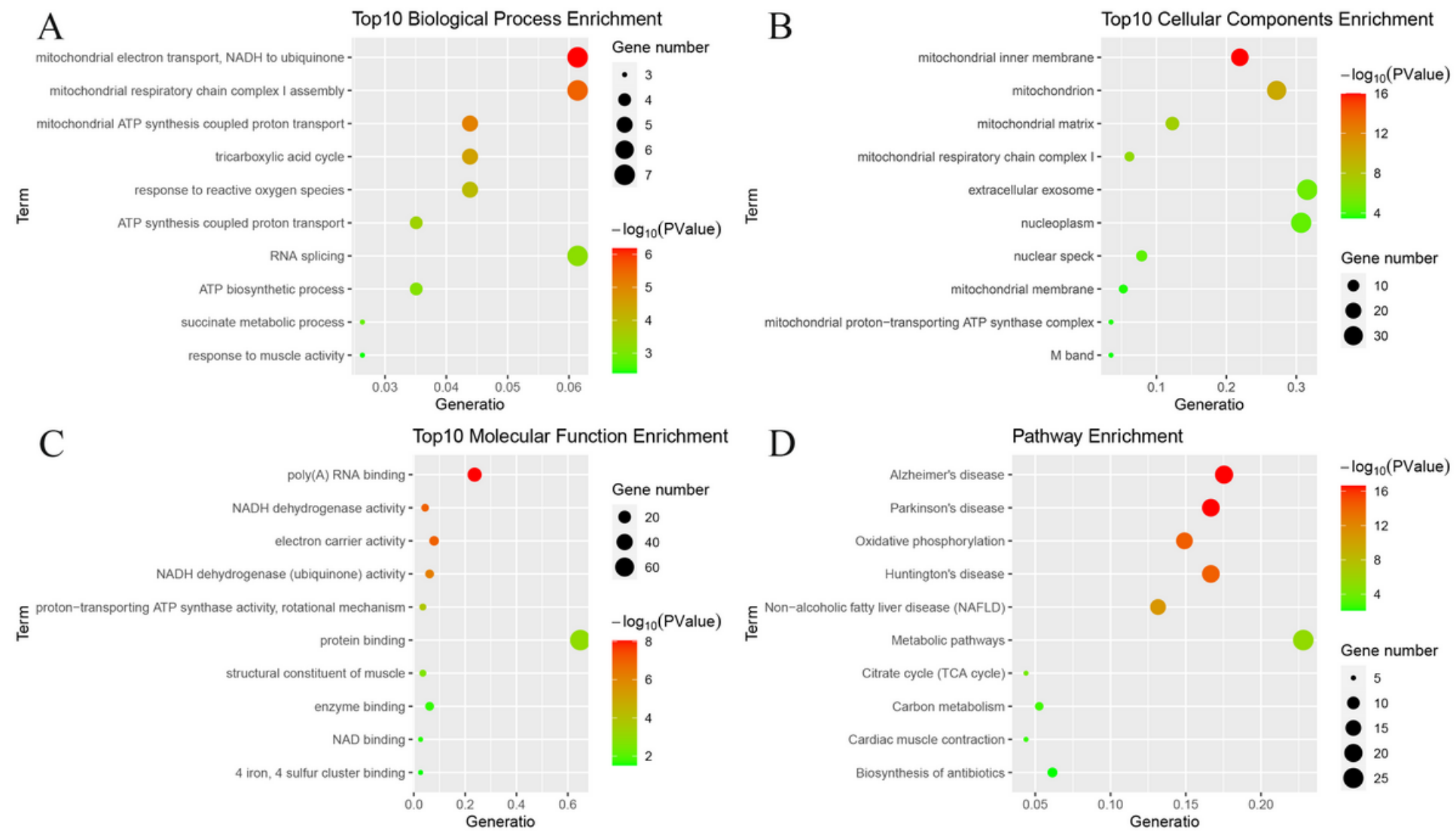

Figure 2

Differentially expression genes enriched in GO and KEGG The color depth of nodes refers to the P-value. The size of nodes refers to the numbers of genes. (A) GO biological process terms. (B) GO cellular components terms. (C) GO molecular function terms. (D) KEGG pathways Abbreviation: GO, gene ontology; KEGG, Kyoto Encyclopedia of Genes and Genomes. 

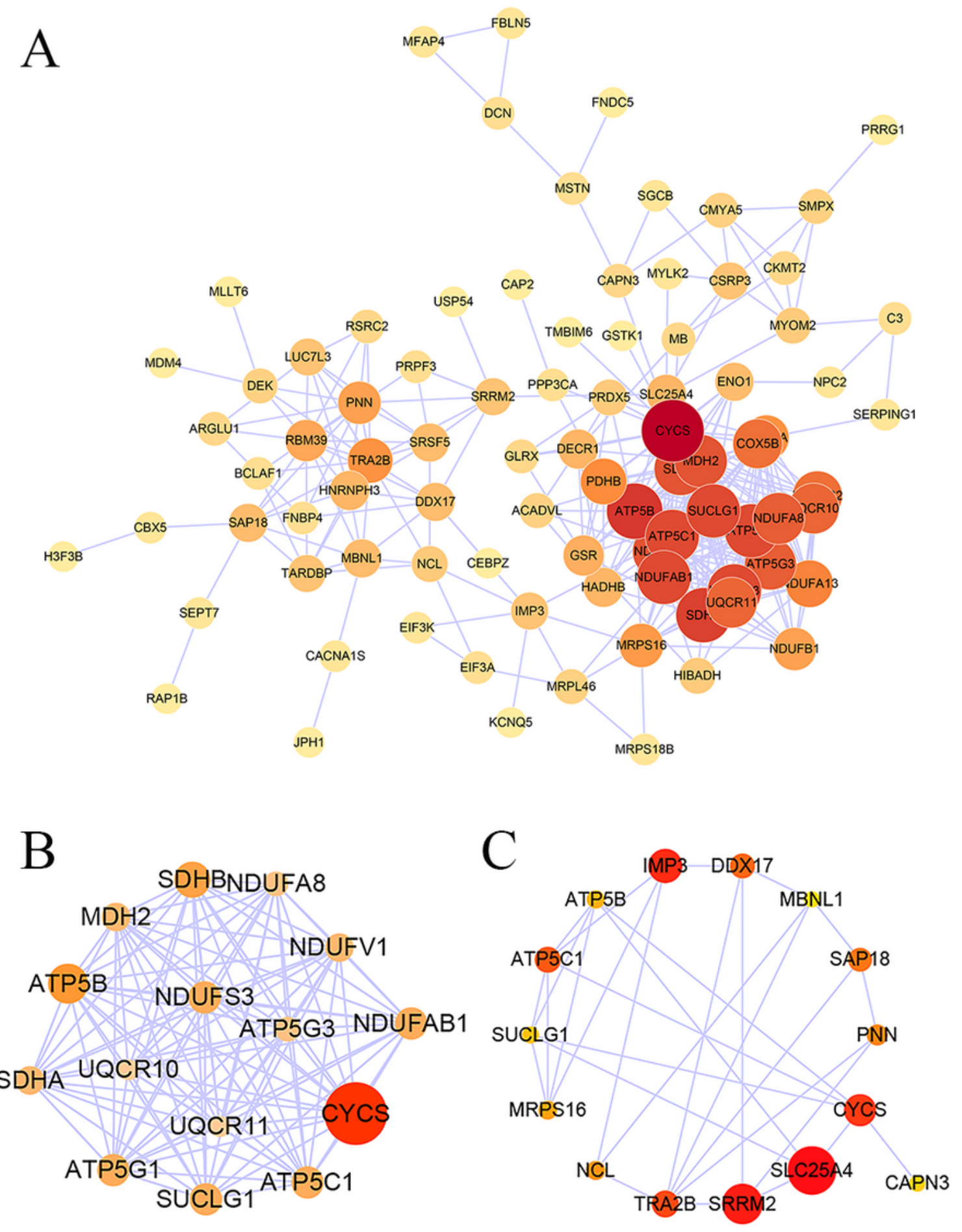

\section{Figure 3}

Protein-protein interaction network and the top 15 genes of degree and stress (A) PPI network of 113 DEGs and 363 edges. (B) The top 15 genes of degree. (C) The top 15 genes of stress. The larger the circle and the redder the color indicates higher the degree or stress. 

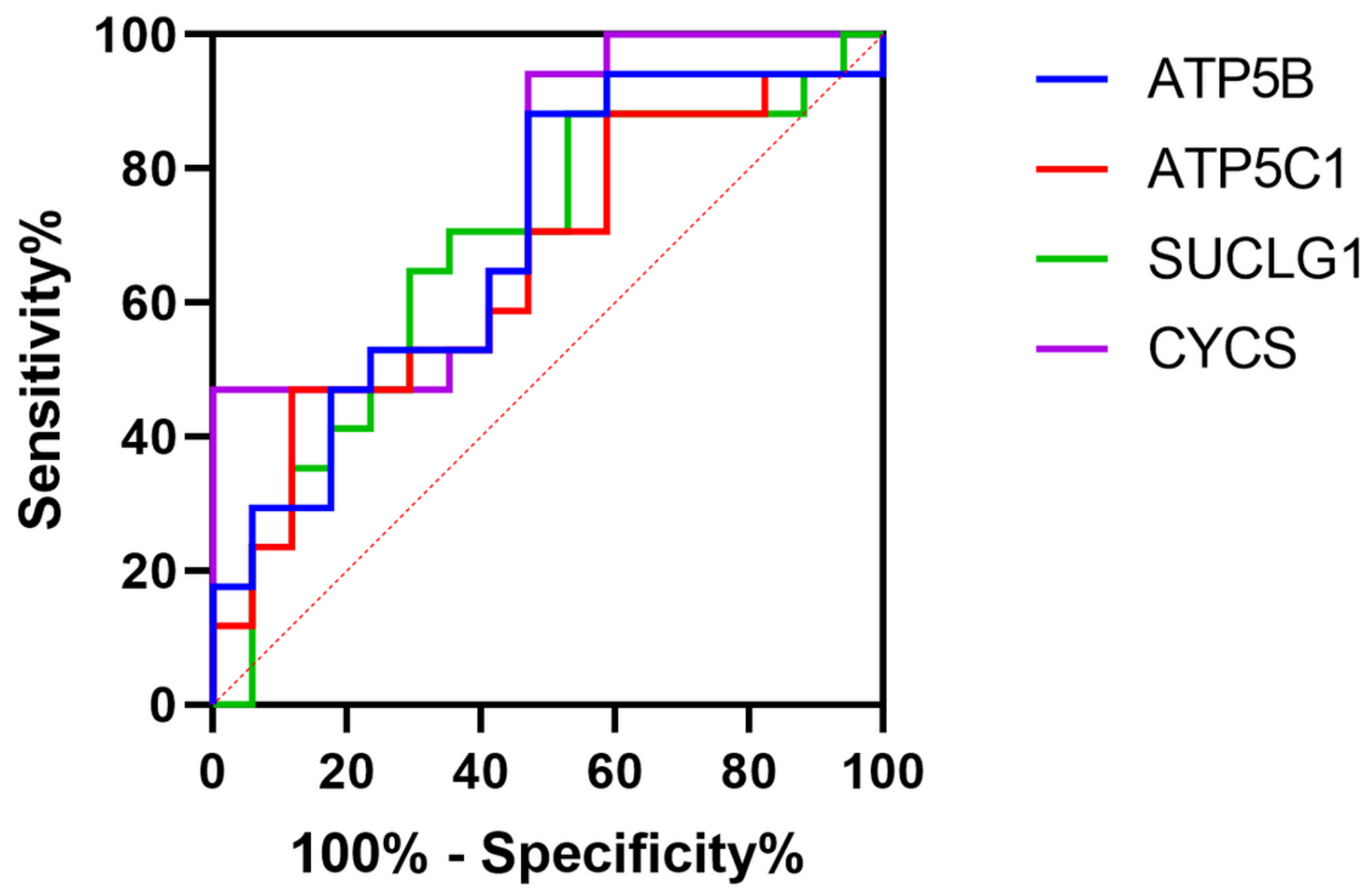

Figure 4

The ROC curve of hub genes 


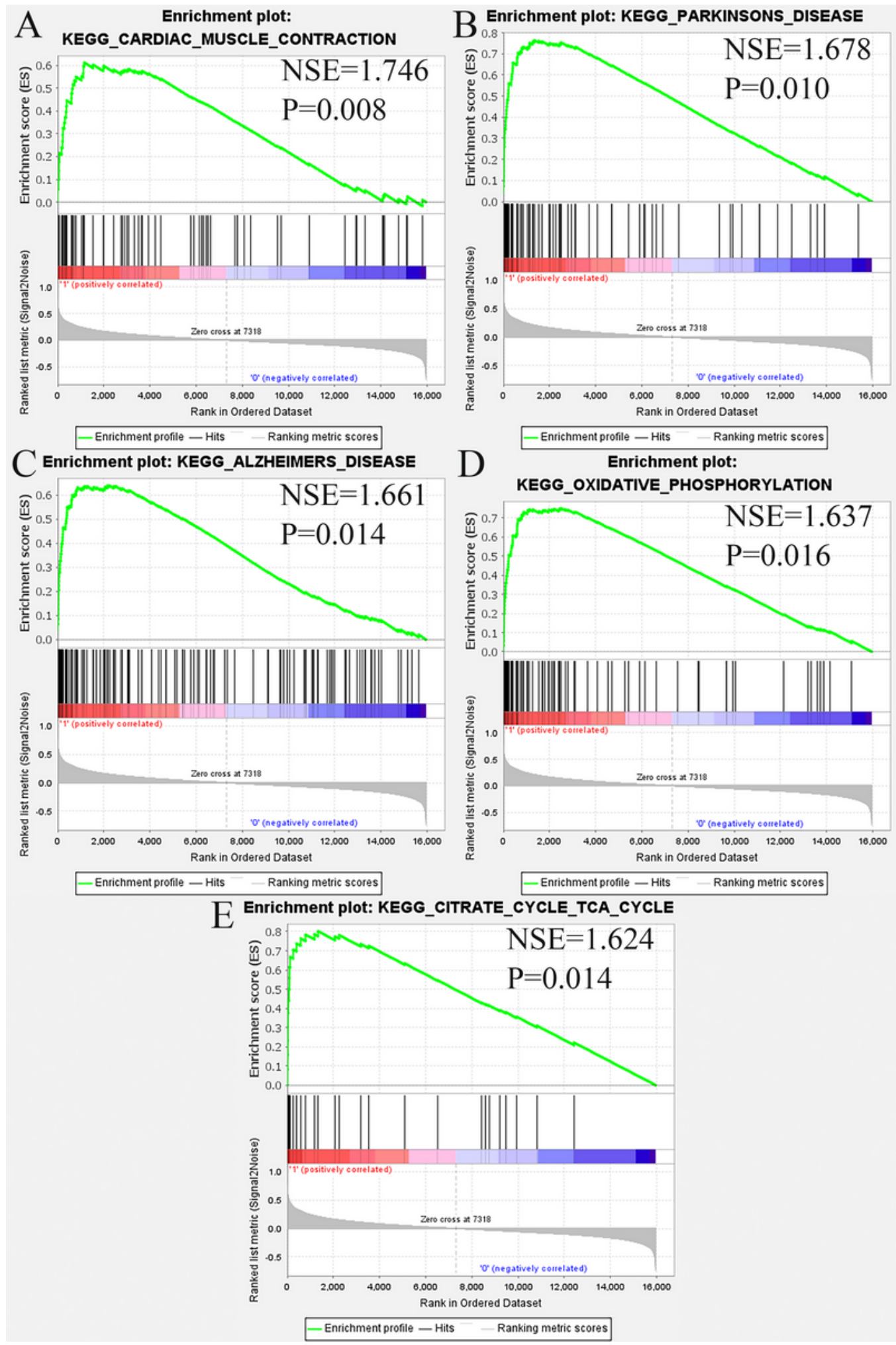

Figure 5

Gene set enrichment analysis of the high expression of CYCS High expression samples of the CYCS were enriched in (A) cardiac muscle contraction, (B) Parkinson's disease, (C) Alzheimer's disease, (D) oxidative phosphorylation, (E) citrate acid cycle (TCA cycle). 